PROCEEDINGS OF THE

AMERICAN MATHEMATICAL SOCIETY

Volume 134, Number 2, Pages 435-442

S 0002-9939(05)08146-3

Article electronically published on June 14, 2005

\title{
CONTRACTION RATIOS FOR GRAPH-DIRECTED ITERATED CONSTRUCTIONS
}

\author{
MANAV DAS
}

(Communicated by Michael Handel)

\begin{abstract}
We provide necessary and sufficient conditions for a graph-directed iterated function system to be strictly contracting.
\end{abstract}

\section{INTRODUCTION}

Let $G=(V, E)$ be a directed graph. For $u, v \in V$ let $E_{u v}$ denote the set of all edges from $u$ to $v$. In the context of iterated function systems, a directed graph is called a Mauldin-Williams graph. An iterated function system realizing the graph is made up of metric spaces $\left(X_{v}, \rho_{v}\right)$, one for each vertex $v \in V$, and similarities $S_{e}$, one for each edge $e \in E$, such that $S_{e}: X_{v} \rightarrow X_{u}$ if $e \in E_{u v}$, and $S_{e}$ has ratio $r_{e}$. An invariant list for such an iterated function system is a list of nonempty compact sets $K_{v} \subseteq X_{v}$, one for each vertex $v$ such that

$$
K_{u}=\bigcup_{v \in V} \bigcup_{e \in E_{u v}} S_{e}\left(K_{v}\right)
$$

for all $u \in V$.

Graph-directed iterated function systems and graph self-similar sets are generalizations of iterated function systems and self-similar sets. Such constructions have been studied by Mauldin and Williams [17, Wang [20, Edgar and Mauldin 7], Olsen [18, Das 11, Strichartz [19, Lau et al. 14], Edgar and Golds 6], Das and Edgar [2], 3], Das and Ngai 4, Haeseler et al. [11] and McClure [16, among others. The following result is well known:

Theorem 0.1 ([5]). Let $G=(V, E, r)$ be a Mauldin-Williams graph. Suppose $r_{e}<1$ for all $e \in E$. Let $\left(S_{e}\right)_{e \in E}$ realize the graph in complete metric spaces $X_{v}$. Then there is a unique list $\left(K_{v}\right)_{v \in V}$ of nonempty compact sets with $K_{v} \subseteq X_{v}$ such that

$$
K_{u}=\bigcup_{v \in V} \bigcup_{e \in E_{u v}} S_{e}\left(K_{v}\right)
$$

for all $u \in V$.

Received by the editors September 13, 2004.

2000 Mathematics Subject Classification. Primary 28A78, 28A80.

Key words and phrases. Directed graphs, graph-directed iterated function systems, self-similar.

(C)2005 American Mathematical Society Reverts to public domain 28 years from publication 
Oftentimes, we have $r_{e}>1$ for some $e \in E$. In such cases, it is important to rescale the construction to make the ratios strictly less than 1 . One may replace each of the metrics $\rho_{v}$ by a constant multiple $\rho_{v}^{\prime}=f_{v} \rho_{v}$ of itself. This affects the similarity ratios in the following manner: if $e \in E_{u v}$, then for $x, y \in X_{v}$,

$$
\rho_{u}^{\prime}\left(S_{e}(x), S_{e}(y)\right)=f_{u} \rho_{u}\left(S_{e}(x), S_{e}(y)\right)=f_{u} r_{e} \rho_{v}(x, y)=\frac{f_{u}}{f_{v}} r_{e} \rho_{v}^{\prime}(x, y) .
$$

The maps $S_{e}$ therefore realize a Mauldin-Williams graph $\left(V, E, r^{\prime}\right)$ where

$$
r_{e}^{\prime}=\frac{f_{u}}{f_{v}} r_{e} \text { for } e \in E_{u v} .
$$

The Mauldin-Williams graph $\left(V, E, r^{\prime}\right)$ is called a rescaling of $(V, E, r)$ and is called contracting iff $r_{e}^{\prime}<1$ for every $e \in E$. Theorem 0.1 shows that a realization of a contracting graph in complete metric spaces has a unique invariant list. It is therefore important to understand when such a rescaling is possible. These considerations motivate the following definitions and the problem.

An edge function is a function $R: E \rightarrow(0, \infty)$. The graph $G$ is strictly contracting with edge function $R$ if and only if $R(e)<1$ for every $e \in E$. A vertex function is a function $f: V \rightarrow(0, \infty)$. Let $R_{f}: E \rightarrow(0, \infty)$ be defined as $R_{f}(e)=\frac{f_{u}}{f_{v}} R(e)$ where $e \in E_{u v}$. It is always possible to find edge functions that would make $G$ strictly contracting.

This raises the following question: Given a graph $G$ and an edge function $R$, does there exist a vertex function $f$ such that $G$ is strictly contracting with edge function $R_{f}$ ?

It is clear that if the graph is already strictly contracting, then the choice $f \equiv$ 1 suffices. It is also clear that a necessary condition for a graph to be strictly contracting is that $R(e)<1$ for every loop, where $e$ is a loop at vertex $v$ means $e \in E_{v v}$.

Let $E^{n}$ denote the set of paths in $G$ that are of length $n$ and $E^{*}=\bigcup_{n=1}^{\infty} E^{n}$. A cycle is a directed graph $H$ for which there is a closed path which passes into every vertex exactly once and such that every edge of $H$ is an edge of this path, we will say that a cycle has length $k$ if it is in $E^{k}$. If $\alpha=e_{1} e_{2} \ldots e_{k}$ is a path in $G$, let $R(\alpha)=R\left(e_{1}\right) R\left(e_{2}\right) \ldots R\left(e_{k}\right)$. For $\alpha \in E^{*}, v_{j} \in V$, we will denote $R(\alpha), f\left(v_{j}\right)$ by $r_{\alpha}, f_{j}$ respectively. Let $G=(V, E, r)$ denote a digraph with edge function $r$. For $\mathbf{x}^{T}=\left(x_{1}, x_{2}, \ldots, x_{k}\right) \in \mathbf{R}^{k}$ we will say $\mathbf{x}>0$ if $x_{i}>0$ for every $i$; similarly $\mathbf{x}<0$ will mean every component is strictly negative. Let $A_{G}$ denote the associated matrix (to be defined in the next section). We provide the following characterization for a graph to be strictly contracting.

Theorem 0.2. Let $G=(V, E, r)$ be a digraph with at least one nonempty cycle. Let $A_{G}$ be the matrix associated to $G$. The following are equivalent:

(1) $G$ is strictly contracting;

(2) $\exists \mathbf{x} \in \mathbf{R}^{n}, \mathbf{x}>0$, such that $A_{G} \mathbf{x}<0$ in $\mathbf{R}^{m}$, where $n=|V|$ and $m=|E|$;

(3) $r_{\alpha}<1$ for every nonempty cycle $\alpha$ in $G$;

(4) $\exists N$ such that $r_{\tau}<1$ for every $\tau \in E^{k}, k \geq N$.

The proof involves some elementary linear algebra. The result provides an immediate solution to the problem of when a graph-directed construction is strictly contracting. It also provides a solution to [5, Exercise 4.3.9]. 
Corollary 0.3. The following conditions are necessary and sufficient for a MauldinWilliams graph $G=(V, E, r)$ to be contracting:

(1) $\exists \mathbf{x} \in \mathbf{R}^{n}, \mathbf{x}>0$, such that $A_{G} \mathbf{x}<0$ in $\mathbf{R}^{m}$, where $n=|V|$ and $m=|E|$;

(2) $r_{\alpha}<1$ for every nonempty cycle $\alpha$ in $G$;

(3) $\exists N$ such that $r_{\tau}<1$ for every $\tau \in E^{k}, k \geq N$.

\section{The ASSOCIATED MATRIX}

Recall that if $e$ is a loop, then $r_{e}<1$ and for any vertex function $f$, we still have $R_{f}(e)=r_{e}<1$. Suppose $e_{1}, e_{2}$ are two edges in $E_{u v}$, and assume $r_{1} \geq r_{2}$. We will let $r_{i}$ denote $r_{e_{i}}$. If $f$ is a vertex function such that $R_{f}\left(e_{1}\right)<1$, then

$$
R_{f}\left(e_{2}\right)=\frac{f_{u}}{f_{v}} r_{2} \leq \frac{f_{u}}{f_{v}} r_{1}=R_{f}\left(e_{1}\right)<1 .
$$

This motivates us to define a reduced graph $\bar{G}$ as follows: it has the same vertex set as $G$ and to obtain the edge set

(1) we delete all the loops;

(2) for each pair of vertices $u, v$ we keep one edge $e \in E_{u v}$ such that $r_{e}=$ $\max \left\{r_{i}: e_{i} \in E_{u v}\right\}$ and delete all other edges. If there is more than one such edge, we will pick one arbitrarily.

By our earlier remarks, it is clear that if the problem can be solved for $\bar{G}$, then it can be solved for any graph that has $\bar{G}$ as its reduced graph. Let $\mathcal{G}$ denote the class of all finite graphs that have at least one cycle of length $\geq 2$. For $G=$ $(V, E, r), G^{\prime}=\left(V, E^{\prime}, r^{\prime}\right) \in \mathcal{G}$, we let $G \sim G^{\prime}$ if and only if they have the same reduced graph. Then $\sim$ is an equivalence relation on $\mathcal{G}$. Without loss of generality, we may henceforth assume that $G$ is a graph that satisfies the following:

G1. it has at least one cycle of length $\geq 2$;

G2. it has no loops, i.e. $E_{u u}=\emptyset$ for every $u \in V$;

G3. for $u, v \in V, E_{u v}$ is either a singleton or empty.

Let $G=(V, E, r)$ with vertex set $V=\left\{v_{1}, v_{2}, \ldots, v_{n}\right\}$, edge set $E=\left\{e_{1}, e_{2}, \ldots\right.$, $\left.e_{m}\right\}$ and the edge function $r$. It follows that $m \geq n \geq 2$. For $v \in V$ we let $\operatorname{out}(v)=\bigcup_{u} E_{v u}$, and $i n(v)=\bigcup_{u} E_{u v}$. To each graph $G$ we associate the $m \times n$ matrix $A_{G}=\left(a_{i j}\right)$ whose columns and rows are indexed by $V$ and $E$ respectively. The components are therefore of the form $a_{i j}=a_{e_{i} v_{j}}$ where

$$
a_{i j}= \begin{cases}r_{i} & \text { if } e_{i} \in \operatorname{out}\left(v_{j}\right), \\ -1 & \text { if } e_{i} \in \operatorname{in}\left(v_{j}\right) .\end{cases}
$$

Note that the choice of this matrix depends on the particular ordering of $V$ and $E$. Also note that $A_{G}$ maps $\mathbf{R}^{n}$ to $\mathbf{R}^{m}$.

We begin with a proposition that contains the main idea for the general case. It also provides us with an algorithm to compute the vertex function in terms of the edge function. Let $\mathbf{e}_{i}^{(k)}=[0 \ldots 1 \ldots 0]^{T}$ denote the $i$-th standard unit vector in $\mathbf{R}^{k}$.

Proposition 1.1. Let $G$ be a cycle $\alpha=\left(e_{1}, e_{2}, \ldots, e_{k}\right)$, where $e_{i}$ is an edge from vertex $v_{i}$ to $v_{i+1}$ and $k \geq 2$. Then

(1) If $r_{\alpha}<1$, then for every $\mathbf{y} \in \mathbf{R}^{k}, \mathbf{y}<0$, there exists $\mathbf{x} \in \mathbf{R}^{k}, \mathbf{x}>0$ such that $A_{G} \mathbf{x}=\mathbf{y}$.

(2) There exists a vertex function $f$ such that $G$ is strictly contracting with edge function $R_{f}$. 
Proof. Note that

$$
A_{G}=\left(\begin{array}{cccccc}
r_{1} & -1 & 0 & \ldots & 0 & 0 \\
0 & 0 & 0 & \ldots & r_{k-1} & -1 \\
-1 & 0 & 0 & \ldots & 0 & r_{k}
\end{array}\right) .
$$

In this proof we will denote $A_{G}$ by $A=\left(a_{i j}\right)$. From elementary linear algebra, it follows that $\operatorname{det}(A)=r_{\alpha}-1$, which is negative since $r_{\alpha}<1$. Moreover for each $i, j$, the cofactor $A_{i j}$ of $a_{i j}$ is positive. Thus by Cramer's rule, the solution to the equation $A \mathbf{x}_{j}=-\mathbf{e}_{j}$ is given by $\mathbf{x}_{j}=\left[x_{1}^{j}, x_{2}^{j}, \ldots, x_{k}^{j}\right]^{T}$ where

$$
x_{i}^{j}=\frac{-A_{j i}}{\operatorname{det}(A)}>0
$$

for each $i, j$. Therefore for each $j=1, \ldots, k$, there exists $\mathbf{x}_{j}>0$ such that $A \mathbf{x}_{j}=\mathbf{e}_{j}$. It follows that for each $\mathbf{y}<0$ there exists $\mathbf{x}>0$ such that $A \mathbf{x}=\mathbf{y}$.

Choose any $\mathbf{x}>0$ such that $A \mathbf{x}<0$ and suppose $\mathbf{x}^{T}=\left(x_{1}, x_{2}, \ldots, x_{k}\right) \in \mathbf{R}^{k}$. Let $e \in E$ be an edge from $v_{i}$ to $v_{j}$. From the construction of $A$ it follows that $-x_{j}+r_{e} x_{i}<0$. Therefore

$$
\frac{x_{i}}{x_{j}} r_{e}<1
$$

for every $e \in E$. Now define the vertex function $f$ as $f\left(v_{i}\right)=x_{i}$. It follows from Equation (1.2) that $R_{f}(e)<1$ for every $e \in E$. This completes the proof of the proposition.

The solution set of the system $A_{G} \mathbf{x}<0, \mathbf{x}>0$, is the region bounded by $k$ hyperplanes in $\mathbf{R}^{k}$ passing through the origin. The conclusion in Proposition 1.1 will follow from a more general result (Theorem 1.4). First a definition: an $m \times n$ matrix $A=\left(a_{i j}\right), m \geq n$, will be called a reflection matrix if

$$
a_{i j}= \begin{cases}-1 & \text { if } j=i \\ 1 & \text { if } j=i-1 \\ \leq 0 & \text { if } i>n, \\ -1 & \text { for some } j, \text { if } i>n \\ 0 & \text { otherwise. }\end{cases}
$$

The terminology will be clear from Theorem 1.4 We will first complete our discussion of the associated matrices.

Lemma 1.2. Let $G$ be a strongly connected digraph with at least one cycle of length $\geq 2$. If $r_{\alpha}<1$ for every nonempty cycle $\alpha$ in $G$, then $A_{G}$ is row equivalent to a reflection matrix where the row equivalence is given by the following elementary transformations:

(1) interchange two rows: $R_{i} \leftrightarrow R_{j}$;

(2) $R_{j} \longleftarrow a_{i} R_{i}+R_{j}$, where $a_{i}>0$.

Proof. Since $G$ is strongly connected, for each vertex $v_{i}$ there exists an edge $e_{j} \in$ $\operatorname{out}\left(v_{i}\right)$. Therefore in $A_{G}$, the entry $a_{i j}=-1$. By interchanging rows, if necessary, we may arrange to have $a_{i i}=-1$ for $i=1, \ldots, n$.

Fix $i$. The edge $e_{i}$ must belong to some cycle. Using the rows corresponding to the edges of one such cycle, we may apply row transformations as in the proof of Proposition 1.1 to obtain a reflection matrix. Note that this is possible since $r_{\alpha}<1$ for every cycle $\alpha$. 
Lemma 1.3. Let $G$ be a digraph with at least one cycle of length $\geq 2$ and no isolated vertices. Let $\left\{H_{1}, H_{2}, \ldots, H_{s}\right\}$ be the strongly connected components of $G$. If $r_{\alpha}<1$ for every nonempty cycle $\alpha$ in $G$, then $A_{G}$ is row equivalent to a matrix of the form:

$$
A=\left(\begin{array}{cccccc}
A_{1} & 0 & 0 & \ldots & 0 & 0 \\
0 & A_{2} & 0 & \ldots & 0 & 0 \\
\vdots & \vdots & \vdots & \vdots & \vdots & \vdots \\
0 & 0 & 0 & \ldots & 0 & A_{s} \\
\star & \star & \star & \ldots & \star & \star \\
\vdots & \vdots & \vdots & \vdots & \vdots & \vdots \\
\star & \star & \star & \ldots & \star & \star
\end{array}\right)
$$

where $A_{i}$ is the row reduced reflection matrix for the associated matrix $A_{H_{i}}$ for the strongly connected component $H_{i}$, each $\star$ is non-positive with at least one negative entry in each row and the row equivalence is given by the following elementary transformations:

(1) interchange two rows: $R_{i} \leftrightarrow R_{j}$,

(2) $R_{j} \longleftarrow a_{i} R_{i}+R_{j}$, where $a_{i}>0$.

Proof. Let $\left\{H_{1}, H_{2}, \ldots, H_{s}\right\}$ be the strongly connected components of $G$. Let $A_{H_{i}}$ be the associated matrix for the strongly connected component $H_{i}$. By Lemma 1.2, we may reduce this to a reflection matrix. Since the strongly connected components are disjoint, we obtain a block diagonal form of the matrix. If these components cover $G$, then we are done. If not, the extra edges give rise to new rows in the matrix. Each such row contains two entries: -1 and $r_{j}$ for some $j$. We may perform row operations to change the entry $r_{j}$ to 0 , and since $r_{\alpha}<1$ for every cycle, these row operations would change the entry -1 to another strictly negative entry.

Theorem 1.4. Let $A$ be an $m \times n$ reflection matrix, where $m \geq n \geq 2$.

(1) If $m=n$, then for every $\mathbf{y} \in \mathbf{R}^{m}, \mathbf{y}<0$, there exists $\mathbf{x} \in \mathbf{R}^{n}, \mathbf{x}>0$, such that $A \mathbf{x}=\mathbf{y}$.

(2) If $m>n$, then there exists $\mathbf{x} \in \mathbf{R}^{n}, \mathbf{x}>0$, such that $A \mathbf{x}<0$.

Moreover, these properties hold for any matrix $A^{\prime}$ that can be reduced to a reflection matrix through elementary transformations of the kind:

(1) interchange two rows: $R_{i} \leftrightarrow R_{j}$;

(2) interchange two columns: $C_{i} \leftrightarrow C_{j}$;

(3) $R_{j} \longleftarrow a_{i} R_{i}+R_{j}$, where $a_{i}>0$.

Proof. If $m=n$, then $A$ is a lower triangular matrix of the form:

$$
A=\left(\begin{array}{cccccc}
-1 & 0 & 0 & \ldots & 0 & 0 \\
1 & -1 & & \ldots & 0 & 0 \\
\vdots & \vdots & \vdots & \vdots & \vdots & \vdots \\
0 & 0 & 0 & \ldots & -1 & 0 \\
0 & 0 & 0 & \ldots & 1 & -1
\end{array}\right)
$$

Let $\mathbf{x}_{i}=\left[x_{1}^{i}, x_{2}^{i}, \ldots, x_{n}^{i}\right]$ where for each $j=1, \ldots, n$,

$$
x_{j}^{i}= \begin{cases}1 & \text { if } j \geq i, \\ 0 & \text { otherwise. }\end{cases}
$$


These are clearly linearly independent vectors in $\mathbf{R}^{n}$ and $A \mathbf{x}_{i}=-\mathbf{e}_{i}$ for $i=1, \ldots, n$.

Suppose $A^{\prime}$ can be reduced to a reflection matrix $A$ by interchanging the $i$-th and $j$-th rows (resp. columns). Let $\mathbf{z}>0$ such that $A \mathbf{z}<0$. Obtain $\mathbf{z}^{\prime}$ by interchanging the $i$-th and $j$-th entries of $\mathbf{z}$. Then $A^{\prime} \mathbf{z}^{\prime}<0$ while $\mathbf{z}^{\prime}>0$.

Suppose $A^{\prime}$ can be reduced to a reflection matrix $A$ by a transformation of the type $R_{j} \longleftarrow a_{i} R_{i}+R_{j}$ where $a_{i}>0$. Then

$$
A^{\prime} \mathbf{z}_{i}=-\mathbf{e}_{i}
$$

has a solution if and only if

$$
A \mathbf{z}_{i}=-\mathbf{e}_{i}-a_{i} \mathbf{e}_{j}
$$

has a solution. So letting

$$
\mathbf{z}_{i}=\mathbf{x}_{i}+a_{i} \mathbf{x}_{j}
$$

gives us the required vectors.

For $m>n$ we consider the $n \times n$ submatrix $A^{\prime}$ of $A$ consisting of rows $R_{1}, \ldots, R_{n}$. This is lower triangular and therefore by the preceding paragraph, we may find $\mathbf{z} \in \mathbf{R}^{n}, \mathbf{z}>0$, such that $A^{\prime} \mathbf{z}<0$ in $\mathbf{R}^{n}$. For $i>n$, the rows $R_{i}$ of $A$ contain at least one strictly negative entry, and all nonpositive entries. Moreover $\mathbf{z}>0$. It follows therefore that $A \mathbf{z}<0$ in $\mathbf{R}^{m}$.

Corollary 1.5. Let $G$ be a digraph with at least one cycle of length $\geq 2$. If $r_{\alpha}<1$ for every nonempty cycle $\alpha$ in $G$, then there exists $\mathbf{x} \in \mathbf{R}^{n}, \mathbf{x}>0$, such that $A_{G} \mathbf{x}<0$.

Proof. Using Lemma 1.3, $A_{G}$ can be row reduced to a reflection matrix, by interchanging rows if necessary. The result is now immediate from Theorem 1.4 .

\section{Proof OF the MAIN RESUlt}

Proof of Theorem 0.2. (1) $\Rightarrow(3)$ : This is obvious.

$(3) \Rightarrow(2)$ : This is the content of Corollary 1.5 .

$(2) \Rightarrow(1)$ : Choose any $\mathbf{x}>0$ such that $A_{G} \mathbf{x}<0$ and suppose $\mathbf{x}^{T}=\left(x_{1}, x_{2}, \ldots\right.$, $\left.x_{n}\right) \in \mathbf{R}^{n}$. Let $e \in E$ be an edge from $v_{i}$ to $v_{j}$. From the construction of $A_{G}$ it follows that $-x_{j}+r_{e} x_{i}<0$. Therefore

$$
\frac{x_{i}}{x_{j}} r_{e}<1
$$

for every $e \in E$. Now define the vertex function $f$ as $f\left(v_{i}\right)=x_{i}$. It follows from Equation (2.1) that $R_{f}(e)<1$ for every $e \in E$. Therefore $G$ is strictly contracting with edge function $R_{f}$.

$(3) \Rightarrow(4)$ : We present the argument in [17, Lemma II]. Let $\alpha=\left(e_{1}, e_{2}, \ldots, e_{l}\right)$ be any cycle in $G$. Let

$$
\Gamma:=\sup \left\{\left(r_{\alpha}\right)^{1 /\left|v_{\alpha}\right|}: \alpha \text { is a cycle in } G\right\},
$$

where $\left|v_{\alpha}\right|=l$ is the number of vertices in the cycle $\alpha$. By assumption $r_{\alpha}<1$ for every nonempty cycle, and therefore $\Gamma<1$. Let $|V|=n$, and choose $\tau=$ $\left(\tau_{1}, \tau_{2}, \ldots, \tau_{k}\right) \in E^{k}, k>n$. By the pigeon-hole principle some index is repeated. Choose a cycle in $\tau$ and factor the product over the cycle from the entire product, and remove the cycle from $\tau$ except for one of the repeated indices. We obtain a 
new path in $G$. Continue as before. For each cycle $\alpha$ there is a nonnegative integer $q_{\alpha}$ such that $r_{\tau}=\prod_{\alpha} r_{\alpha}^{q_{\alpha}} r_{\beta}$, where $\beta \in E^{s}$ for some $s \leq n$. Therefore

$$
r_{\tau}=\prod_{\alpha}\left[\left(r_{\alpha}\right)^{1 /\left|v_{\alpha}\right|}\right]^{\left|v_{\alpha}\right| q_{\alpha}} r_{\beta} \leq \Gamma^{\sum\left|v_{\alpha}\right| q_{\alpha}} r_{\beta}
$$

Taking the $k$-th root of both sides and letting $k$ go to infinity, we have

$$
\lim _{k \rightarrow \infty}\left[\sup _{\mathbf{e} \in E^{k}} r_{\mathbf{e}}\right]^{1 / k} \leq \Gamma<1 .
$$

This gives us the existence of $N$.

$(4) \Rightarrow(3)$ : Suppose there exists a cycle $\alpha$ in $G$ such that $r_{\alpha} \geq 1$. Clearly $\alpha$ must have length $l<N$. So there exists $k$ such that $k l \geq N$. Let $\tau=\underbrace{\alpha \alpha \ldots \alpha}_{k \text { times }}$. Since $\alpha$ is a cycle, $\tau \in E^{k l}$, and $r_{\tau}=r_{\alpha}^{k} \geq 1$, which contradicts (4) since $k l \geq N$. This completes the proof of the theorem.

\section{REFERENCES}

1. M. Das, Hausdorff measures, dimensions and mutual singularity, Trans. Amer. Math. Soc., (to appear).

2. M. Das and G. A. Edgar, Separation properties for graph-directed self-similar fractals, Topology Appl., (to appear).

3. M. Das and G. A. Edgar, (preprint). Weak separation in graph-directed self-similar fractals.

4. M. Das and S.-M. Ngai, Graph-directed iterated function systems with overlaps, Indiana Univ. Math. J. 53 (2004), 109-134. MR.2048186

5. G. A. Edgar, Measure, topology, and fractal geometry, Springer-Verlag, New York, 1990. MR 1065392 (92a:54001)

6. G. A. Edgar and Jeffrey Golds, A fractal dimension estimate for a graph-directed iterated function system of non-similarities, Indiana Univ. Math. J. 48 (1999), no. 2, 429-447. MR 1722803 (2001b:28012)

7. G. A. Edgar and R. D. Mauldin, Multifractal decompositions of digraph recursive fractals, Proc. London Math. Soc. 65 (1992), 604-628. MR.1182103 (93h:28010)

8. K. J. Falconer, Techniques in fractal geometry. John Wiley \& Sons, Ltd., Chichester, 1997. MR.1449135 (99f:28013)

9. K. J. Falconer, Fractal geometry. Mathematical foundations and applications, John Wiley \& Sons, Ltd., Chichester, 1990. MR.1102677 (92j:28008)

10. J. E. Hutchinson, Fractals and self-similarity, Indiana Univ. Math. J. 30 (1981), 713-747. MR $0625600(82 \mathrm{~h}: 49026)$

11. Fritz v. Haeseler, Heinz-Otto Peitgen and Gencho Skordev, Global analysis of self-similarity features of cellular automata: selected examples, Phys. D 86 (1995), no. 1-2, 64-80. MR 1353952 (97b:58075)

12. Su Hua and Hui Rao, Graph-directed structures of self-similar sets with overlaps, Chinese Ann. Math. Ser. B 21 (2000), no. 4, 403-412. MR1801772 (2003g:11087)

13. I. N. Herstein and David J. Winter, Matrix theory and linear algebra. Macmillan Publishing Company, New York, 1988. MR0999134 (90h:15001)

14. K.-S. Lau, J. Wang and C.-H. Chu, Vector-valued Choquet-Deny theorem, renewal equation and self-similar measures, Studia Math. 117 (1995), 1-28. MR1367690 (97m:43001)

15. M. McClure, Directed-Graph Iterated Function Systems, Mathematica in Education and Research, (to appear).

16. Mark McClure, The Hausdorff dimension of Hilbert's coordinate functions, Real Anal. Exchange 24 (1998/99), no. 2, 875-883. MR1704763(2000h:26013)

17. R. D. Mauldin and S. C. Williams, Hausdorff dimension in graph directed constructions, Trans. Amer. Math. Soc., 309, (1988), 811-829. MR0961615(89i:28003) 
18. Lars Olsen, Random Geometrically Graph Directed Self-Similar Multifractals, Pitman Research Notes in Mathematics Series, Vol. 307, Longman Scientific and Technical, 1994. MR 1297123 (95j:28006)

19. R. S. Strichartz, Self-similar measures and their Fourier transforms III, Indiana Univ. Math. J., 42, (1993), 367-411. MR1237052(94j:42025)

20. JingLing Wang, The open set conditions for graph directed self-similar sets, Random Comput. Dynam., 5, (1997), no. 4, 283-305. MR1483871 (99g:28019)

21. Hui Xu, The Hausdorff dimension and measure of conformal graph-directed sets, (Chinese) Acta Math. Sinica, 44, (2001), no. 4, 633-640. MR1850164(2002h:28008)

Department of Mathematics, 328 Natural Sciences Bullding, University of LouisVille, Louisville, KentudKy 40292 